\title{
Metabolism of the bovine cumulus-oocyte complex and influence on subsequent developmental competence
}

\author{
JG Thompson, M Lane and RB Gilchrist \\ Research Centre for Reproductive Health, School of Paediatrics and Reproductive Health, The \\ University of Adelaide, Adelaide, 5005
}

\begin{abstract}
The two types of cells that make up the cumulus-oocyte complex (i.e. the oocyte and cumulus cells) have very different metabolic demands, with glucose occupying a central role in metabolic activity. Cumulus cells have a significant requirement for and utilise high levels of glucose, yet appear to have little need for oxidative metabolism. In contrast, oocytes have a requirement for oxidative metabolism, although limited glucose metabolism may also be an important aspect of meiotic and developmental competence. Nevertheless, because of the metabolic and communication link between the cumulus and the oocyte, glucose availability and metabolism within the cumulus can have a significant impact on oocyte meiotic and developmental competence. In particular, the role of the hexosamine biosynthesis pathway within cumulus cells appears critical for the supply of substrate from glucose for extracellular matrix production, yet if overstimulated can significantly decrease developmental competence of the oocyte. Current static systems for in vitro maturation are clearly incompatible with meeting substrate demands, especially glucose. In the future, in vitro maturation will include a more dynamic approach, which will adjust nutrient components to meet the changing functional requirements of cumulus-oocyte complexes during the final process of maturation.
\end{abstract}

\section{Introduction}

Understanding oocyte meiotic and developmental competence and unravelling the factors that mediate their fulfilment remain as great challenges in the field of reproductive biology, although recent attempts to describe the molecular basis of oocyte competence have taken significant steps forward (Pan et al. 2005; Reis et al. 2006). In contrast, the benefits that will result from a greater understanding of oocyte meiotic and developmental competence have not altered. We are still inept in our ability to exploit fully the genetic value held within every female gamete, which could be far greater by capturing the significant numbers of oocytes within an individual female, each capable of producing a viable offspring. We are also still inept in our ability to store oocytes in any developmental state so as to efficiently derive viable embryos when needed. 


\section{Oocyte developmental competence and oocyte capacitation}

Oocyte developmental competence is the term that refers to the biochemical and molecular state that allows a mature oocyte to support development of the embryo and ultimately a healthy offspring at term. We still have a very poor understanding of what constitutes oocyte developmental competence, including the role that the environment surrounding an oocyte plays in its progress towards acquisition of developmental competence. The difficulty of studying the oocyte within the developing follicle remains the major barrier to rapid progress in this area. It is clear that oocytes gradually and sequentially acquire meiotic and developmental competence during the course of folliculogenesis. During the early stages of antral follicle growth, non-rodent oocytes are actively synthesizing RNA and as antral follicles progress towards ovulatory size, the synthetic activity of the oocyte is gradually reduced until the oocyte reaches a quiescent state (de Smedt et al. 1994; Fair et al. 1995). This latter phase of oocyte development has been termed "oocyte capacitation" (Hytell et al. 1997) as it is during this phase of oogenesis that the oocyte acquires the cytoplasmic machinery necessary to fully support preimplantation embryo development (Brevini-Gandolfi \& Gandolfi 2001). The somatic cells of the follicle, and the cumulus cells in particular, certainly play a key role in the acquisition of developmental competence in vivo. However, we have only a limited understanding of the nature and diversity of compounds that transfer between the cumulus cells and the oocyte via gap junctions during this final phase of follicular development (Albertini et al. 2001) and we still have no idea of how dynamic this process is and whether or not dynamic changes to levels of transferring molecules impacts the process of developmental competence. We also have a poor understanding of the role that granulosa and cumulus cells have in modifying the extracellular environment that surrounds the oocyte; speculation with little data is usually the norm here (e.g. nutrients) and little consideration is taken for the influence of events such as gap junction breakdown and cumulus expansion have on nutrient availability to the oocyte.

Our laboratory has taken an interest in the metabolism of the cumulus-oocyte complex (COC) while undergoing in vitro maturation (IVM) and what role this may have on oocyte developmental competence, especially in the bovine. It is noteworthy that oocyte IVM involves the artificial removal of oocytes, generally from mid-sized antral follicles, that presumably have not completed oocyte capacitation. Incubating COCs in vitro leads effectively to a precocious spontaneous meiotic maturation, that occurs in the absence of these crucial events and environments that are required for complete cytoplasmic maturation of the oocyte. Many may argue that this is a poor model for studying the far more complex interactions that take place within the follicle during final antral follicle development. Whilst this is reasonable, the fact remains that oocyte IVM technologies, especially in domestic animals, must deal with the very mixed population of oocytes collected from follicles at varying stages of development and atresia. Our efforts are directed at trying to understand the complex metabolic interplay between oocytes and cumulus cells as they undergo maturation in vitro, in an attempt to develop IVM systems that actually confer developmental competence on oocytes that would otherwise undergo natural demise in vivo by subordination and atresia.

Here we review the progress we, and others, have made in our understanding of the metabolic conditions that influence both meiotic and developmental competence during IVM of bovine COCs in recent times. Other reviews in the area that precede this and may be of useful reference include Sutton et al. (2003b) and Krisher (2004). 


\section{Energy demands by the oocyte - developmental competence}

Mammalian oocytes are essentially reliant on oxidative phosphorylation for the generation of ATP (Rieger and Loskutoff 1994; Steeves and Gardner 1999). Not surprisingly, the level of ATP within a mature oocyte appears now as a key indicator of developmental competence (Hashimoto et al. 2000a; Stojkovic et al. 2001). Van Blerkom (2004) has long argued the importance of mitochondria within human oocytes in the development of competence. This has only recently been examined in several other species, e.g. mouse (Thouas et al. 2004) and bovine (Stojkovic et al. 2001: Tarazona et al. 2006), which are all in agreement on the importance of mitochondria. However, there is also evidence that the activity of the glycolytic pathway within the oocyte following maturation also relates to developmental competence (Krisher and Bavister 1999; Steeves and Gardner 1999), despite the low levels of glycolytic activity within bovine oocytes (Rieger and Loskutoff 1994; Cetica et al. 2002).

\section{Concentration of substrates in follicular fluid}

Several recent publications have described levels of energy substrates available to immature, compact ruminant COCs from antral follicles prior to the LH surge (Table 1). Concentrations of glucose and pyruvate have been measured at approximately $2-3 \mathrm{mM}$ and $0.4 \mathrm{mM}$ respectively in these studies. Although studies have found lactic acid is also present within follicular fluid, care must be used in interpreting this, as most data has been derived from ovaries collected in an abattoir in which the time from slaughter to fluid collection will affect lactic acid levels.

Table 1. Glucose and carboxylic acid concentrations (Mean \pm S.E.) in follicular fluid from various sizes of follicles $($ small $=\sim 3 \mathrm{~mm}$, medium $=-5 \mathrm{~mm}$, large $=\sim 8 \mathrm{~mm})$

\begin{tabular}{|c|c|c|c|c|}
\hline & Small & Medium & Large & \\
\hline Glucose (mM) & $\begin{array}{l}2.01 \pm 0.10 \\
1.4 \pm 0.2 \\
3.5 \pm 0.5\end{array}$ & $\begin{array}{c}2.85 \pm 0.16 \\
2.2 \pm 0.3 \\
-\end{array}$ & $\begin{array}{l}3.75 \pm 0.18 \\
2.3 \pm 0.2 \\
3.9 \pm 0.4\end{array}$ & $\begin{array}{l}\text { Leroy et al. (2004) } \\
\text { Sutton McDowall et al. (2005) } \\
\text { Iwata et al. (2004). }\end{array}$ \\
\hline Pyruvate (mM) & $0.4 \pm 0,1$ & $0.4 \pm 0.1$ & $0.4 \pm 0.0$ & $\begin{array}{lll}n \\
n \\
n\end{array}$ \\
\hline Lactate (mM) & $\begin{aligned} & 14.4 \pm 0.35 \\
& 3.0 \pm 0.7 \\
&-\end{aligned}$ & $\begin{array}{c}9.4 \pm 0.35 \\
6.4 \pm 1.7 \\
-\end{array}$ & $\begin{array}{l}5.6 \pm 0.37 \\
3.2 \pm 0.6\end{array}$ & " \\
\hline
\end{tabular}

Less is known regarding other substrates. Recently, Leroy et al. (2004) measured, amongst other parameters, levels of $B$-hydroxybutyrate, urea and non-esterified fatty acids within bovine follicular fluid. Berg and colleagues (Berg et al. 2003) have reported that $\mathrm{O}_{2}$ concentrations within dominant follicles in vivo are around $67-86 \mathrm{mmHg}$, (i.e. around $9-12 \% \mathrm{O}_{2}$ ), which seems higher than many other species (reviewed by Sutton et al. 2003b).

\section{The uptake of oxygen by bovine COCs}

Although oxygenation of the follicle has been linked with oocyte developmental competence, (Van Blerkom 1998; Berg et al. 2003), there is little known regarding the oxygen consumption by intact COCs from any mammalian species and what role this plays in oocyte growth and 
development. Sutton and colleagues (Sutton et al. 2003a) measured oxygen uptake by bovine COCs over the course of a $24 \mathrm{~h}$ in vitro maturation period (in the presence of FSH). On a per ng DNA basis, COCs increase their oxygen consumption from approximately $50 \mathrm{pmol} / \mathrm{h}$ at the start of maturation, when the COC is compact, to $90 \mathrm{pmol} / \mathrm{h}$ for fully expanded COCs, which equates to from around 1.5 to $2.5 \mathrm{nl} / \mathrm{COC} / \mathrm{h}$. This total uptake of oxygen is remarkably low for a complex of cells such as the COC and is comparable to about double the respiration rate of a bovine blastocyst $(0.9 \mathrm{nl} / \mathrm{h}$, Thompson et al. 1996). Mouse COCs consume an average of $4 \mathrm{nl} /$ $\mathrm{h} \mathrm{O}_{2}$ during maturation (Downs et al. 1997), which is higher, but within a similar range as the bovine COC. These values led us to ask what level of $\mathrm{O}_{2}$ actually reaches the oocyte within the cumulus complex? In particular, we wanted to know if there is significant loss of oxygen within the $\mathrm{COC}$ as $\mathrm{O}_{2}$ diffuses towards the oocyte, especially in immature, compact COCs. We developed a mathematical model (Clark et al. 2006), which assumes the COC is spherical, but accounts for a non-linear consumption of oxygen dependent on the external concentration. Our model also accounts for the two layers of cells, the cumulus layer and the oocyte and differs from other such models (e.g. Byatt-Smith et al. 1991). The model revealed that, surprisingly, the loss of oxygen across the compact bovine COC is negligible (less than $4 \%$ of the external concentration), even if hypoxic levels of oxygen are utilised. Thus the level that reaches the oocyte is effectively that which surrounds the COC in the follicular antrum. This has led us to postulate that the structure and metabolic activity of antral follicles is such that follicular oxygen is preserved for the oocyte, as oocytes are dependent on oxidative phosphorylation for ATP production (Rieger and Loskutoff 1994) and have little capacity for glycolysis (Cetica et al. 2002).

\section{The central role and importance of glucose to oocyte meiotic and developmental competence}

\section{Uptake of glucose by the COC}

The bovine COC from antral follicles consumes substantial quantities of glucose - 23 times more uptake on a per volume basis compared to oocytes (Thompson et al. 2005), pointing to the importance of glucose to COC metabolism (Fig. 1). Indeed, under standard IVM conditions (TCM199 medium containing $5.6 \mathrm{mM}$ glucose), where the COC density is $1: 5 \mu$ l medium, we have shown that over $70 \%$ of glucose will be metabolised and this may well impact glucose uptake and metabolism kinetics (Sutton-McDowall et al. 2004). Over the course of FSHstimulated in vitro maturation, the uptake rate in TCM199-based medium is initially $23 \mathrm{pmol} /$ ngDNA/h, increasing to $40 \mathrm{pmol} / \mathrm{ngDNA} / \mathrm{h}$. This is entirely due to uptake by the cumulus cells, as the bovine oocyte itself has a very low capacity for glucose uptake (Rieger and Loskutoff 1994; Zuelke and Brackett 1992). There is evidence that ruminant granulosa cells contain both GLUT1 and the insulin-sensitive GLUT4, which may explain the high uptake rates (Williams et al. 2001).

The induction of cumulus expansion increases glucose uptake by approximately $25 \%$ and is associated with an increase in hyaluronic acid synthesis via the hexosamine biosynthesis pathway (Sutton-McDowall et al. 2004). Using a non-metabolised fluorescent glucose analogue, we have observed the temporal uptake of glucose within the immature compact and fully expanded COC (Thompson et al. 2005). Although preliminary at this stage, in immature COCs, the non-metabolised glucose forms a gradient of fluorescence through the cumulus cell layers towards the oocyte. Within 40-60 min of exposure, significant accumulation occurs within the corona radiata. From approximately $40 \mathrm{~min}$, fluorescence is observed within the oocyte. As this is a non-metabolised glucose, the question that remains to be answered is: Does this reflect the transfer of unmetabolised glucose or some metabolite of glucose? How much actual glu- 


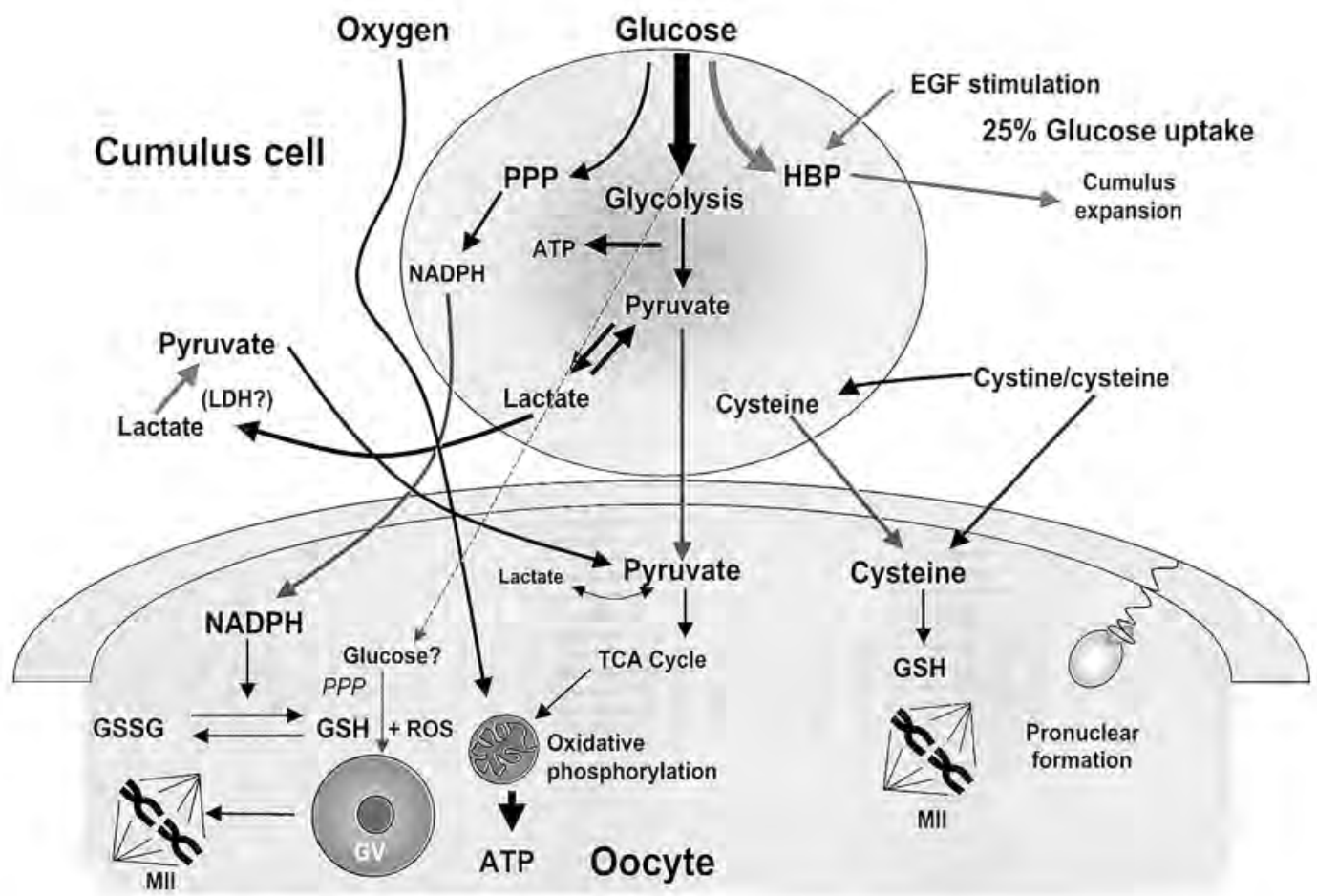

Fig. 1, Schematic representation of the metabolism of glucose and carboxylic acids, plus the amino acid cysteine in the cumulus oocyte complex during maturation. Direction of arrows represents the direction of substrate transfer and density of arrows suggests possible activity of pathways. Colours of arrows (other than black) represent stage of maturation, with blue specific for substrate transfer occurring prior to gap junction breakdown and red specific for activity following cumulus expansion. Abbreviations: ATP = Adenosine triphosphate; EGF = Epidermal Growth Factor; GSH = glutathione (reduced); GSSG glutathione (oxidised); GV - Germinal vesicle; HBP = Hexosamine biosynthesis pathway $\mathbf{L D H}=$ Lactate dehydrogenase $; \mathrm{MII}=$ Metaphase II; PPP $=$ Pentose Phosphate Pathway; $\mathrm{NADPH}=$ Nicotinamide adenine dinucleotide phosphate; $\mathrm{ROS}=$ reactive oxygen species.

cose reaches the oocyte within the immature compact cumulus complex? To answer this, we are developing a much more sophisticated mathematical model to understand the dynamics of facilitated transport, diffusion and metabolism across entities such as the COC. Nevertheless, Saito and others (Saito et al. 1994) have shown that in the mouse both glucose and glucose-6phosphate levels are high in the immature oocyte derived from preovulatory follicles, falling dramatically with (presumably) the loss of cumulus cell gap-junction connection, strongly suggesting that prior to gap junction breakdown, both glucose and glucose metabolites are transferred from cumulus cells into the oocyte. In expanded COCs, over the same time course, it is clear that cumulus cells play little role in glucose transport into the oocyte as no fluorescence appears to enter the oocyte, similarly to that described in the mouse (Saito et al. 1994). This further supports the work of others that glucose uptake is very low in mature bovine oocytes (Rieger and Loskutoff 1994). 
The effect of glucose on meiotic and developmental competence of the bovine oocyte

Several studies have demonstrated that the presence of glucose during spontaneous in vitro maturation is necessary for complete meiotic maturation to occur (Hashimoto et al. 2000b). However, recent studies have revealed that glucose availability (i.e. concentration vs. depletion as a result of uptake over the course of maturation) can have significant affects on the progression of maturation (Iwata et al. 2004; Sutton-McDowall et al. 2005). Indeed, the use of sequential IVM systems, where fresh medium is used to "replenish" glucose levels during the maturation, can alleviate poor meiotic responses in media with low levels of glucose. Nevertheless, delayed maturation appears to have little consequence in terms of developmental competence (Iwata et al. 2004; Sutton-McDowall et al. 2006). In contrast, also using a sequential maturation media, Atef and colleagues (Atef et al. 2005) found exposure to FSH for 6 hours during IVM was beneficial to development when using a low $(1.5 \mathrm{mM})$ glucose concentration maturation medium (Synthetic Oviduct Fluid) compared with continuous exposure (no change in medium) or $2 \mathrm{~h}$ of FSH (and exchange at that time). We suggest these latter two treatments also induce significant glucose depletion at the volume per COC that was utilized ( $5 \mu \mathrm{l} / \mathrm{COC}$ ). Depletion of glucose may prevent oocytes from maintaining glucose-6-phosphate levels (Saito et al. 1994), which is known to have a stimulatory effect on meiotic progression via activity of the pentose phosphate pathway (Downs et al. 1998).

\section{The hexosamine biosynthesis pathway and oocyte competence}

In most somatic cells the hexosamine biosynthesis pathway (HBP, Fig. 2) is a minor glucose metabolic pathway, accounting for 1-3\% of glucose metabolism (Marshall et al. 1991). A major role of the HBP is the generation of $\mathrm{N}$-linked acetyl sugars for the production of glycosaminoglycans. For example, the end product of the HBP is UDP-N-acetylglucosamine (UDP-N-GlcNAc), which is then synthesized into hyaluronic acid by the action of the enzyme, hyaluronic acid synthase (Fig. 2). The HBP is considered a self-regulating pathway; the rate-limiting enzyme is also the first step of the pathway, glutamine-fructose-6-phosphate transaminase (GFPT). Inhibition of GFPT activity is regulated by the pathway's end product, UDP-N-GlcNAC (McKnight et al. 1992). Cumulus cells have a great capacity for hyaluronic acid synthesis as a result of initiation of expansion by epidermal growth factor like factors in vivo (Park et al. 2004) or by FSH/EGF in vitro. As previously mentioned, glucose uptake increases $25 \%$ with the initiation of cumulus expansion (Sutton-McDowall et al. 2004), which appears to be entirely due to up regulation of the HBP pathway. How such a dramatic increase in activity is initiated remains to be determined, but it appears that factors regulating cumulus expansion do so by regulating GFPT activity.

An alternate fate for UDP-N-GICNAC is O-linked glycosylation, via O-linked glycosyltransferase, an X-linked enzyme (O'Donnell et al. 2004). The importance of O-linked glycosylation within protein signalling systems has only recently come to light, with many major signalling proteins now recognized as being regulated by O-linked glycosylation (e.g. Sp1, Sp3, CREB, p53, in addition to many others). In particular, serines and threonines that are normally phosphorylated can also be O-linked glycosylated, thereby changing the activity (either up or down-regulated) of phosphorylated signalling proteins (Wells et al. 2003; Zachara and Hart 2004). One of the more widely studied effects of HBP up-regulation leading to increased O-linked glycosylation is insulin signalling through the insulin receptor substrate-1 (Andreozzi et al. 2004), where perturbed phosphorylation of the insulin receptor substrate significantly down-regulates the protein kinase B pathway.

Glucosamine is a sugar related to glucose and can be transported into cells by members of the facilitated glucose transporter family, similarly to glucose (albeit with different kinetics). However, unlike glucose, there is only one metabolic fate for glucosamine, phosphorylation to 

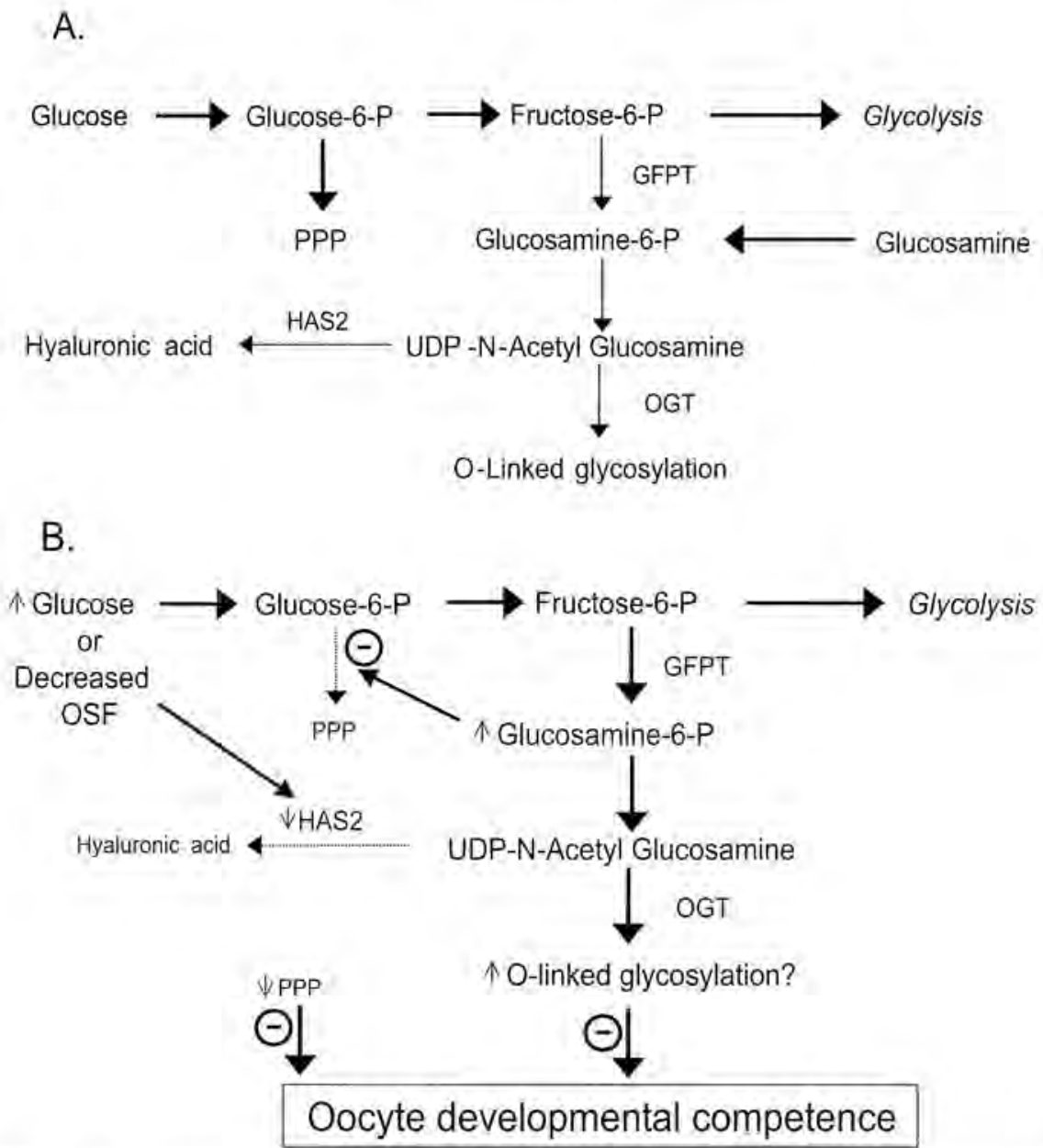

Fig. 2. A. Glucose metabolism, with particular focus on the hexosamine biosynthesis pathway. B. The effect of reduction of oocyte secreted factors or hyperglycaemia on developmental competence. An increase in the hexosamine biosynthesis pathway activity or diversion of UDP-N-acetylglucosamine towards O-linked glycosylation (either by hyperglycaemia or by inhibition of cumulus expansion) causes a reduction in developmental competence of the oocyte. Abbreviations: GFPT = glutamine:fructose-6-phosphate transaminase; HAS2 = hyaluronic acid synthase; OSF = oocyte secreted factors; PPP $=$ Pentose phosphate pathway.

glucosamine-6-phosphate (Fig. 2) and thus bypasses the rate-limiting enzyme of the hexosamine pathway. The consequence is that UDP-N-GICNAC levels increase significantly, which can lead to increased O-linked glycosylation. Glucosamine is therefore regarded as a hyperglycaemic mimetic.

Glucosamine addition to a bovine IVM medium significantly reduced the level of glucose incorporated within the expanding cumulus mass (Sutton-McDowall et al. 2004), signifying the role the hexosamine pathway plays in extracellular matrix formation. Surprisingly, although not significantly affecting meiotic maturation or early cleavage rates following fertilization, glucosamine addition during IVM also substantially reduced subsequent developmental com- 
petence (Sutton-McDowall et al. 2006), with almost complete inhibition at levels of $2.5 \mathrm{mM}$ glucosamine (when incubated with $5.6 \mathrm{mM}$ glucose). This loss of developmental competence was somewhat reversed when inhibition of O-linked glycosyltransferase in the presence of glucosamine was performed (Sutton-McDowall et al. 2006). However, we have not yet fully determined if the glucosamine effect is mediated through cumulus cell signalling, or if it has a direct affect on the oocyte, although immunohistochemical staining of O-linked glycosylation was largely isolated to within cumulus cells, indicating the former rather than the latter.

Hashimoto and others (Hashimoto et al. 2000b) have also found that under hyperglycaemic conditions, developmental competence was compromised. This fits well with our model (Fig. 2B), where either hyperglycaemic conditions or compromised cumulus expansion mechanisms leads to an inhibition of developmental competence. However, this inhibition was overcome and, furthermore, developmental competence improved when oxygen concentration was reduced from atmospheric levels $\left(20.9 \%\right.$ ) to $5 \% \mathrm{O}_{2}$ (Hashimoto et al. 2000a). Indeed, only when hyperglycaemic conditions were utilized, was low oxygen found to benefit developmental competence (Hashimoto et al. 2000a). This interaction between oxygen and glucose may possibly be explained by the involvement of the transcription factor, hypoxia inducible factor (HIF). Lowering $\mathrm{pO}_{2}$ may activate $\mathrm{HIF}$ to increase glycolytic enzyme activities, which are regulated by HIF (lyer et al. 1998), increasing the capacity to metabolise glucose via glycolysis, thus reducing the option for glucose to be utilised for the HBP and O-linked glycosylation (see Fig. 2B). HIF is also believed to mediate selected FSH-induced responses (Alam et al. 2004), so that low oxygen levels may increase the effectiveness of FSH within the maturation system.

\section{Role of carboxylic acids}

Most of the glucose taken up by an immature bovine COC is accounted for by the production of lactic acid by cumulus cells and efflux into the surrounding environment (Sutton et al. 2003a). Initially in IVM, a positive correlation exists between lactic acid production and glucose uptake, which then weakens as maturation proceeds (Sutton et al. 2003a). This, as well as the unchanging lactic acid production over the course of FSH-stimulated cumulus expansion and glucose uptake, provides further evidence that the increasing glucose uptake that occurs during maturation is directed into pathways other than glycolysis (Sutton et al. 2003a). Exogenous pyruvate uptake by the FSH stimulated bovine COC occurs, even in the presence of glucose (Sutton et al. 2003a). A role for exogenous pyruvate, however, is largely unknown, especially in the presence of adequate glucose. Glycolysis is particularly active in cumulus cells and they have significant levels of several isoforms of lactate dehydrogenase (LDH, the close to equilibrium enzyme for pyruvate conversion to lactate) (Cetica et al. 1999; 2002). Therefore it remains to be shown that there is a need for exogenous pyruvate, at least for much of the first nine hours of bovine IVM, where gap-junction communication is patent (Thomas et al. 2004). On the other hand, one can imagine that following initiation of cumulus expansion (and hence rapid loss of the oocyte's source of glycolytic metabolites), direct exposure of the oocyte to adequate levels of pyruvate for the latter period of maturation could be necessary for the oocyte. Interestingly, exogenous pyruvate appeared to have no influence on developmental competence, whereas exogenous lactate did (Rose-Hellekant et al. 1998), One possible explanation may be the high levels of lactate present in the medium as maturation proceeds and the release of LDH from apoptotic cumulus cells, especially those on the outer edge of the COC (Hussein et al. 2005), providing a source of pyruvate for the oocyte towards the end of maturation. 


\section{Amino acids}

The role of cysteine (and related sulphated amino acids, such as cysteamine), along with glutamine and proline as substrates for glutathione, is now well characterised as promoting developmental competence in bovine oocytes, and oocytes from other species (de Matos et al. 1995; de Matos and Furnus 2000). Inclusion of these sulphated amino acids during IVM and the subsequent increase in oocyte glutathione levels is thought to reduce the level of reactive oxygen species production within oocytes, which has beneficial effects on subsequent developmental competence (de Matos and Furnus 2000). Consequently, cysteamine is now a standard additive in most IVM culture systems. In addition, glutamine is known to be stimulatory for cumulus expansion (Rose-Hellekant et al. 1998), most likely through its involvement in hyaluronic acid synthesis through the hexosamine biosynthesis pathway (see above). Zuelke and Brackett (1993) also found that under the influence of luteinizing hormone, glutamine oxidation increased within the COC, although the relevance of this observation remains obscure.

\section{Lipids}

Little new work has emerged on the role of lipids during oocyte IVM, with the exception of the efforts of Leroy and co-workers (Leroy et al. 2005), who have an interest in the hypothesis that non-esterified fatty acids within follicular fluid play a role in oocyte competence during periods of negative energy balance in high performing dairy cows. Their recent work has revealed that addition of stearic and palmitic acids, but not oleic acid, had negative affects on oocyte developmental competence when included during IVM (Leroy et al. 2005).

\section{Conclusions}

The bovine cumulus oocyte complex contains two cell types that appear to have antagonistic nutritional demands. Cumulus cells have a great need and appetite for glucose. Too Jittle glucose during maturation appears to have significant adverse effects on oocyte meiotic competence, whilst too much appears to affect oocyte developmental competence. The former may involve glucose and glucose-6-phosphate within the oocyte itself, the latter appears to be mediated through the hexosamine biosynthesis pathway and links the process of supplying substrate for cumulus expansion with oocyte developmental competence. However, achieving consistent glucose concentrations under static IVM conditions is problematic due to the high uptake rate. In contrast, the demand for oxygen and oxidative substrates appear low.

Oocytes, on the other hand, require oxygen and oxidative phosphorylation, and although some glycolytic activity occurs, we question its significance prior to cumulus expansion and loss of gap-junction communication.

The anatomical design of the cumulus oocyte complex fits these antagonistic demands, as oxygen appears to readily diffuse through the cumulus layer to the oocyte, with little being consumed by the cumulus cells themselves. In contrast, glycolytic activity within cumulus cells is such that some glucose appears to reach the immature oocyte whilst the cumulus layer remains compact. This changes once cumulus expansion occurs, so that little glucose enters the oocyte.

Achieving such a delicate balance in substrate supply during oocyte in vitro maturation will remain difficult whilst the current static culture systems employed continue to be utilized. Novel culture systems, utilizing principles of perfusion, microfluidics and small culture chambers with large media reservoirs - the WOW system (Vajta et al. 2000), will facilitate more 
purpose-built conditions that satisfy the complex demands required for successful in vitro maturation of oocytes.

\section{Acknowledgements}

Many thanks to Melanie Sutton-McDowell, Karen Kind and members of the Early Development Group, RCRH, The University of Adelaide for their assistance with this work. Research support for the authors was provided by the Australian Research Council and Cook Australia Pty Ltd, in addition to National Health and Medical Research Council (Australia) and the National Institute of Health (USA).

\section{References}

Alam H, Maizels E T, Park Y, Ghaey S, Feigert Z J, Chandel, N S and Hunzicker-Dunn M 2004 Follicle-stimulating hormone activation of Hypoxia-inducible factor-1 by the phosphatidylinositol 3-Kinase/ AKT/Ras homolog enriched in brain (Rheb)/mammalian target of rapamycin (mTOR) pathway is necessary for induction of select protein markers of follicular differentiation. Journal of Biological Chemistry 279 19431-19440.

Albertini D F, Combelles C M H, Benecchi E and Carabastsos M J 2001 Cellular basis for paracrine regulation of ovarian follicle development. Reproduction 121 647-653.

Andreozzi F, D'Alessandris C, Federici M, Laratta E, Del Guerra S, Del Prato S, Marchetti P, Lauro R, Perticone, $F$ and Sesti G 2004 Activation of the hexosamine pathway leads to phosphorylation of IRS-1 on Ser 307 and Ser612 and impairs the phosphatidylinositol 3 . kinase/Akt/mTOR insulin biosynthetic pathway in RIN pancreatic \{beta\}-cells. Endocrinology $\mathbf{1 4 5}$ 2845-57.

Atef A, Francois P, Christian V and Sirard M-A 2005 The potential role of gap junction communication between cumulus cells and bovine oocytes during in vitro maturation. Molecular Reproduction and Development 71 358-367.

Berg D K, Beaumont SE, Berg M C, Hull C D and Tervit H R 2003 Oxygen and carbon dioxide tension in days 14-15 dominant bovine follicles measured in vivo or 4 hours post-mortem. Theriogenelogy $\mathbf{5 9}$ 406.

Brevini-Gandolfi T A L and Gandolfi F 2001 The materna legacy to the embryo: cytoplasmic components and their effects on early development. Theriogenology 55 1255-1276.

Byatt-Smith I G, Leese H J and Gosden R G 1991 An investigation by mathematical modelling of whether mouse and human preimplantation embryos in static culture can satisfy their demands for oxygen by diffusion. Human Reproduction 6 52-57.

Cetica P, Pintos L, Dalvit G and Beconi M 2002 Activity of key enzymes involved in glucose and triglyceride catabolism during bovine oocytes maturation in vitro Reproduction 124 675-681.

Cetica P D, Pintos L N, Dalvit G C and Beconi M T 1999 Effect of lactate dehydrogenase activity and isoenzyme focalisation in bovine oocytes and utilisation of oxidative phosphorylation on in vitro maturation. Theriogenology 51 541-550.

Clark A R, Stokes Y M, Lane M and Thompson J G 2006 Mathematical modelling of oxygen concentration in bovine and murine cumulus-oocyte complexes. Reproduction 131 999-1006.

de Matos D G and Furnus C C 2000 The importance of having high glutathione (GSH) level after bovine in vitro maturation on embryo development effect of B-mercaptoethanol, cysteine and cystine. Theriogenology 53 761-771.

de Mato, D G, Furnus C C, Moses D F and Baldasarre H 1995 Effect of cysteamine on glutathione level and developmental capacity of bovine oocyte matured in vitro. Molecular Reproduction and Development 42 - $432-436$,

de Smedt V, Crozet N and Gall L 1994 Morphological and functional changes accompanying the acquisition of meiotic competence in ovarian goat oocyte. lournal of Experimental Zoology 269 128-139.

Downs S, Humpherson P and Leese H 1998 Meiotic induction in cumulus cell-enclosed mouse oocytes: Involvement of the Pentose Phosphate Pathway. Biology of Reproduction 58 1084-1094.

Downs S M, Houghton F D, Humpherson P G and Leese H J 1997 Substrate utilization and maturation of Cumulus cell-enclosed mouse oocytes: evidence that pyruvate oxidation does not mediate meiotic induction. Journal of Reproduction and Fertility 110 1-10.

Fair T, Hyttel P and Greve T 1995 Bovine oocyte diameter in relation to maturational competence and transcriptional activity. Molecular Reproduction and Development 42 437.442.

Hashimoto S, Minami N, Takakura R, Yamada M, Imai $\mathrm{H}$ and Kashima N 2000a Low oxygen tension during in vitro maturation is beneficial for supporting the subsequent development of bovine cumulusoocyte complexes. Molecular Reproduction and Development 57 353-360. 
Hashimoto S, Minami N, Yamada M and Imai H 2000b Excessive concentration of glucose during in vitro maturation impairs the developmental competence of bovine oocytes after in vitro fertilisation: Relevance to intracellular reactive oxygen species and glutathione contents, Molecular Reproduction and Development 56 520-526.

Hussein T S, Froiland D A, Amato F, Thompson J G and Gilchrist R B 2005 Oocytes prevent cumulus cell apoptosis by maintaining a morphogenic paracrine gradient of bone morphogenetic proteins. Journal of Cell Science 15 5257-5268.

Hytell P, Fair T, Callesen H and Greve T 1997 Oocyte growth, capacitiation and final maturation in cattle. Theriogenology 47 23-32.

Iwata H, Hashimoto S, Ohota M, Kimura K, Shibano K and Miyake M 2004 Effects of follicle size and electrolytes and glucose in maturation medium on nuclear maturation and developmental competence of bovine oocytes, Reproduction 127 159-164.

Iyer N V, Kotch L E, Agani F, Leung S W, Laughner E, Wenger R H, Gassmann M, Gearhar, I D, Lawler A M, Yu Q Y and Semenza G L 1998 Cellular and developmental control of $\mathrm{O}_{2}$ homeostasis by hypoxiainducible factor 1a. Genes and Development 12 149-162.

Krisher R L 2004 The effect of oocyte quality on development. Journal of Animal Science 82 14-23.

Krisher R L and Bavister B D 1999 Enhanced glycolysis after maturation of bovine oocytes in vitro is associated with increased developmental competence. Molecular Reproduction and Development $\mathbf{5 3} 19$. 26.

Leroy J L M R, Vanholder T, Mateusen B, Christophe A, Opsomer G, de Kruif A, Genicot G and Van Soom A 2005 Non-esterified fatty acids in follicular fluid of dairy cows and their effect on developmental capacity of bovine oocytes in vitro. Reproduction 130 485-495.

Leroy J L M R, Vanholder T R D J, Opsomer G, Van Soom A J B P E and De Kruif A 2004 Metabolite and ionic composition of follicular fluid from differentsized follicles and their relationship to serum concentrations in dairy cows. Animal Reproduction Science 80 201-211.

Marshall D L, Bacote V and Traxinger R R 1991 Discovery of a metabolic pathway mediating glucose-induced desenitization of the glucose transport system. fournal of Biological Chemistry 266, 4706-4712.

McKnight G L, Mudri S L, Mathewes S L, Traxinger R R, Marshall S, Sheppard P O and O'Hara,P 1992 Molecular cloning, cDNA sequence, and bacterial expression of human glutamine:fructose-6-phosphate amidotransferase. Journal of Biological Chemistry $26725208-25212$.

O'Donnell N, Zachara N E, Hart G W and Marth JD 2004 Ogt-Dependent X-Chromosome-Linked Protein Glycosylation is a Requisite Modification in Somatic Cell Function and Embryo Viability. Molecular and Celluarl Biology 24 1680-1690.

Pan $\mathrm{H}$, $\mathrm{O}^{\prime}$ Brien $\mathrm{M}$ J, Wigglesworth K, Eppig J J and
Shultz R M 2005 Transcript profiling during mouse oocyte development and the effect of gonadotropin priming and development in vitro. Developmenta! Biology 286 493-506.

Park J $Y$, Su Y Q, Ariga $M$, Law E, Jin S L and Conti $M$ 2004 EGF-like growth factors as mediators of LH action in the ovulatory follicle. Science 303682 684.

Reis A., Chang HY and Levasseur M J K 2006 APCcdh1 activity in mouse oocytes prevents entry into the first meiotic division. Nature Cell Biology 8.539 . 540 .

Rieger D and Loskutoff N M 1994 Changes in the metabolism of glucose, pyruvate, glutamine and glycine during maturation of cattle oocytes in vitro. lournal of Reproduction and Fertility 100 257-262.

Rose-Hellekant T A, Libersky-Williamson E A and Bavister B D 1998 Energy substrates and amino acids provided during in vitro maturation of bovine oocytes alter acquisition of developmental competence. $Z y$ gote 6 285-294.

Saito T, Hiroi M and Kato T 1994 Development of glucose utilization studied in single oocytes and preimplantation embryos from mice. Biology of Reproduction 50 266-270.

Steeves T E and Gardner D K 1999 Metabolism of Glucose, pyruvate, and glutamine during the maturation of oocytes derived from pre-pubertal and adult cows. Molecular Reproduction and Development 54 92-101.

Stojkovic M, Machado S A, Stojkovic P, Zakhartchenko V, Hutzler P, Goncalves P B and Wolf E 2001 Mitochondrial distribution and adenosine triphosphate content of bovine oocytes before and after in vitro maturation: correlation with morphological criteria and developmental capacity after in vitro fertilization and culture. Biology of Reproduction 64904 909.

Sutton M L, Cetica P D, Beconi M T, Kind K L, Gilchrist R B and Thompson J G 2003a Influence of oocytesecreted factors and culture duration on the metabolic activity of bovine cumulus cell complexes. Reproduction $12627-34$.

Sutton M L, Gilchrist R B and Thompson J G 2003b Effects of in-vivo and in-vitro environments on the metabolism of the cumulus-oocyte complex and its influence on oocyte developmental capacity. Human Reproduction 9 35-48.

Sutton-McDowall M L, Gilchrist R B and Thompson J G 2004 Cumulus expansion and glucose utilisation by bovine cumulus-oocyte complexes during in vitro maturation: the influence of glucosamine and follicle-stimulating hormone. Reproduction 128314 319.

Sutton-McDowall M L, Gilchrist R B and Thompson J G 2005 Effect of hexoses and gonadotrophin supplementation on bovine oocyte nuclear maturation during in vitro maturation in a synthetic follicle fluid medium. Reproduction, Fertility, and Development 17 407-415.

Sutton-McDowall M L, Mitchell M, Cetica P, Dalvit G, 
Pantaleon M, Lane M, Gilchrist R and Thompson J G 2006 Glucosamine supplementation during in vitro maturation inhibits subsequent embryo development: Possible role of the hexosamine pathway as a regulator of developmental competence. Biology of Reproduction 74 881-888.

Tarazona A M, Rodriguez I I, Restrepo L F and OliveraAngel M 2006 Mitochondrial activity, distribution and segregation in bovine oocytes and in embryos produced in vitro. Reproduction in Domestic Animals 41 5-11.

Thomas R E, Thompson I G, Armstrong D T and Gilchrist R B 2004 Effect of specific phosphodiesterase isoenzyme inhibitors during in vitro maturation of bovine oocytes on meiotic and developmental capacity. Biology of Reproduction 71 1141-1149.

Thompson J, Clark A, Froiland D, Stokes $\mathrm{Y}$ and Lane $\mathrm{M}$ $2005 \mathrm{~A}$ "nutrient" sparing hypothesis to explain the nutrition of the immature bovine cumulus oocyte complex. Biology of Reproduction Special Issue 417.

Thompson J G, Partridge R I, Houghton F D, Cox C I and Leese H J 1996 Oxygen uptake and carbohydrate metabolism by in vitro derived bovine embryos. Journal of Reproduction and Fertility 106299 306.

Thouas G A, Trounson A, Wolvetang E J and Jones G M 2004 Mitochondrial dysfunction in mouse oocytes results in preimplantation embryo arrest in vitro. Bjology of Reproduction 71 1936-1942.

Vajta G, Peura T T, Holm P, Paldi A, Greve T, Trounson $\mathrm{AO}$ and Callesen $\mathrm{H} 2000$ New method for culture of zona-included or zona-free embrvos: the well of the well (WOW) system. Molecular Reproduction and Development 55 256-264.

Van Blerkom I 1998 Epigenetic influences in oocyte developmental competence: perifollicular vascularity and intrafollicular oxygen. lournal of Assisted Reproduction and Genetics 15 226-234.

Van Blerkom J 2004 Mitochondria in human oogenesis and preimplantatioin embryogenesis: engines of metabolism, ionic regulation and developmental competence. Reproduction 128 269-280.

Wells L, Whelan S A and Hart G W 2003 O-GlcNAC: a regulatory post-translational modification. Biochemical and Biophysical Research Communications. 302 435-441.

Williams S A, Blache D, Martin G B, Foot R, Blackberry M A and Scaramuzzi R J 2001 Effect of nutritional supplementation on quantities of glucose transporters. 1 and 4 in sheep granulosa and theca cells. Reproduction 122 947-956.

Zachara N E and Hart G W 2004 O-GlAc a sensor of cellular state: the role of nucleocytoplasmic glycosylation in modulating cellular function in response to nutrition and stress. Biochimica et Biophysica Acta 1673 13-28.

Zuelke K A and Brackett B G 1992 Effects of luteinizing hormone on glucose metabolism in cumulus-enclosed bovine oocytes matured in vitro. Endocrinology 131 2690-2696.

Zuelke K A and Brackeft B G 1993 Increased glutamine metabolism in bovine cumulus cell-enclosed and denuded oocytes after in vitro maturation with lutenising hormone, Biology of Reproduction 48 815820. 